\title{
Review on Reformulation of the Mean-Variance Model with Real-life Trading Restrictions
}

\author{
Feng $\mathrm{Li}^{1}$ \\ ${ }^{1}$ School of Economics and Management, Tongji University, Shanghai, China \\ Correspondence: Feng Li, School of Economics and Management, Tongji University, Shanghai 200092, P. R. \\ China. Tel: 181-0195-1376. E-mail: lifeng123@tongji.edu.cn
}

Received: September 11, 2017

Accepted: November 21, 2017

Online Published: December 27, 2017

doi:10.5539/ass.v14n1p40

URL: https://doi.org/10.5539/ass.v14n1p40

\begin{abstract}
In this paper, we consider a class of portfolio selection problems with cardinality and minimum buy-in threshold constraints in real-life which can be formulated as mixed-integer quadratic programming (MIQP). Two reformulation methods that generate the same tight continuous relaxation of original problem are compared in the context under the branch-and-bound algorithm, one is the Perspective Reformulation and another is the Lift-and-Convexification Reformulation (LCR). Computational results show that the (PC) is more competitive than the (LCR) method in terms of computing time and nodes in MIQP solver CPLEX 12.7, what's more, this outperformance becomes more obvious as the size of instances grows.
\end{abstract}

Keywords: Portfolio selection, MIQP, Perspective reformulation, LCR, Branch-and-Bound algorithm

\section{Introduction}

Since Markowitz first proposed the mean-variance model in (Markowitz, 1952), the portfolio selection problem has become a popular research topic in the field of modern financial research. Considering there are $n$ risky assets in the market. The expected return vector and the covariance matrix for the assets are $\mu$ and $Q$ respectively. Supposing investor has amount of money to invest in a certain period of time and he has to decide which risky assets to buy and how to allocate his capitals so that raising the revenue and reducing the risk as much as possible. The resulting investment MV model revealing the problem that of trading returns versus risk which can be formulated as:

$$
\begin{array}{r}
\min x^{T} Q x \\
\text { s.t. } e^{T} x=1, \\
\mu^{T} x \geqslant \rho
\end{array}
$$

where $x=\left(x_{1}, x_{2}, \ldots, x_{n}\right)$ represents the proportion of the investment in risky asset $i, i=1, \ldots, n$, the objective function $x^{T} Q x$ on behalf of the associated risk, constraint (1) depicts a feasible allocation of the available resources over assets, constraint (2) meant that the expected return exceeds prescribed expected return level set by investor.

Motivated by Markowitz's work, many researchers have conducted a series of extension work based on mean-variance model. Ogryczak and Ruszczynski (1997); Ouederni and Sullivan (1991); Green and Hollifield (1992); Mao (1970) measured the risk by semivariance and established a mean-semivariance model of portfolio selection. Markowitz (1959) also proved that the mean-semivariance model has better performance than MV model. Konno and Yamazaki (1991) proposed a mean absolute deviation (MAD) model which can remove the most disadvantages of classical MV model and be solved by linear program with largely reduced computational effort. With the rapid development of computer technology, many scholars turned to investigate the skew model for the reason that skewness plays an important role if the distribution of the rate of assets return is asymmetric around the mean. Konno, Shirakawa and Yamazaki (1993) introduced skewness into the mean-absolute deviation model. Then, Konno and Suzuki (1995) introduced the skewness to the mean-variance model, established the mean-variance-skew model and solved the model by integer programming method quickly and efficiently. Value-at-Risk (VaR) was proposed as a risk measure tool to evaluate the maximum loss of assets at a certain probability level for a specific period of time. When using the VaR, however, the original optimization problem will be transformed into a nonconvex problem which is difficult to solve. To overcome this difficulty, 
Rockafellar and Uryasev proposed an improved risk measurement method in (Rockafellar \& Uryasev, 2000; Uryasev, 2000) based on VaR - Conditional Value at Risk (CVaR). Since then, many scholars have demonstrated that the CVaR method is superior to the VaR method in the risk measurement of various financial optimization problems through empirical analysis, such as Andersson, Mausser, Rosen and Uryasev (2001); Rockafellar and Uryasev (2002); Mansini, Ogryczak and Grazia Speranza (2003); Topaloglou, Vladimirou and Zenios (2002).

Compared with above models, the standard MV problem is a convex quadratic programming and easy to solve. However, in many real cases, rational investor would not completely follow the classical MV model due to many practical factors, for example, a lot of very small holdings will yield extra transaction and management costs, so the minimum and maximum buy-in thresholds can not be negligible in practice with the expression form of $x_{i} \in\{0\} \cup\left[\alpha_{i}, \beta_{i}\right], i=1, \ldots, n$. This type of variable is mathematically called semi-continuous variable. We have another expression of this structure with the binary variable:

$$
\alpha_{\mathrm{i}} y_{i} \leq x_{i} \leq \beta_{i} y_{i}, y_{i} \in\{0,1\}, i=1, \ldots, n
$$

What's more, the MV model often appears in many practical applications along with the cardinality constraint to limit the number of asset to invest. The mathematical expression of cardinality constraints can be formulated as following:

$$
\operatorname{card}(x) \leq K
$$

Where $\operatorname{card}(x)$ explains the number of nonzero variables $x_{i}, K$ is an integer with $1 \leq K \leq n$. Moreover, the cardinality constraints are usually expressed as $e^{T} y \leq K$ with binary variable $y_{i} \in\{0,1\}, i=1, \ldots, n$ when modeling the real-word optimization problems, where $e$ is an vector of all ones. So we focus on the mean-variance portfolio optimization problems with cardinality and minimum buy-in threshold constraints in this paper which can be formulated as following:

$$
\begin{aligned}
& (\mathrm{QP}) \quad \min f(x)=x^{T} Q x+c^{T} x \\
& \text { s.t. } e^{T} x=1, \\
& \mu^{T} x \geqslant \rho \\
& \\
& \alpha_{\mathrm{i}} y_{i} \leq x_{i} \leq \beta_{i} y_{i}, y_{i} \in\{0,1\}, i=1, \ldots, n \\
& e^{T} y \leq K
\end{aligned}
$$

where $Q$ is an $n \times n$ positive semi definite symmetric matrix, $c \in \square^{n}, \mu \in \square^{n}, \rho \in \square^{n}$, constraint (6) is referred as minimum buy-in threshold constraint to prevent the investors from holding some assets with a very small amount and constraint (7) is the cardinality constraint to limit the total number of different assets in the optimal portfolio. With the joint of cardinality and minimum threshold constraints, the original quadratic programming problem turned into a mixed-integer quadratic programming (MIQP) problem with semi-continuous variables. Various solution methods for this class of portfolio optimization problems with cardinality and minimum threshold constrains have been investigated in the literature by many researchers, such as Bertsimas and Shioda (2009); Bienstock (1996); Bonami and Lejeune (2009); Cesarone, Scozzari and Tardella (2009); Cui, Zheng, Zhu and Sun (2013); Gao and Li (2013); Li, Sun and Wang (2006). Many heuristic algorithms also have been used to solve such MV problems such as genetic algorithms, tabu search, andsimulated annealing (see, e.g., (Chang, Meade, Beasley, \& Sharaiha, 2000; Blog, Hoek, \& Timmer, 1983; Jacob, 1974; Maringer \& Kellerer, 2003; Mitra, Ellison, \& Scowcroft, 2007)). Despite the various virtues of heuristic algorithm, it cannot guarantee to find the optimal solution or a satisfactory solution of (QP).

In general, problem (QP) is NP-hard problem. The difficulty of finding an efficient solution for this class of MIQP problems arises from the discrete structure induced by the semi-continuous variables in the model so that it has become an active area of research. Typical solutions for this class of MIQP are based on branch-and-bound algorithm. Thus estimating a tighter lower bound which can substantially improve the efficiency of the branch-and-bound algorithm is of much importance. In real applications, however, the lower bound generated by the standard continuous relaxation is too loose to improve the computation efficiency. Therefore many equivalent reformulations have been proposed for the tighter lower bound.

Perspective reformulation is a well-performed reformulation method since it depends on replacing the original convex function in the formulation with its so-called perspective function which is related to the convex envelope of the objective function in original problem. Frangioni and Gentile added the perspective cuts to the reformulation which objective function is either separable in (Frangioni \& Gentile, 2006) or nonseparable in (Frangioni, \& Gentile, 2007). Four types reformulations of tractable perspective relaxation have been proposed to solve the high nonlinearity of objective function due to the fractional term in perspective function. Frangioni 
and Gentile (2009) compared two of them, one is the second-order cone program (SOCP) reformulation, another is perspective cuts $(\mathrm{PC})$ reformulation, and then a new method called projected perspective relaxation $\left(\mathrm{P}^{2} \mathrm{R}\right)$ had been put up in (Frangioni, Gentile, Grande, \& Pacifici, 2011) under three further restrict assumptions so that the perspective relaxation of MIQP can be reformulated as a piecewise linear-quadratic problem and then the consequent model can be simplified as roughly the same size of the original standard continuous relaxation. However, this method has many limitations in application. To solve it, Frangioni, Furini, \& Gentile (2016) invented another $\mathrm{P}^{2} \mathrm{R}$ based approach-Approximated Projected Perspective Reformulation $\left(\mathrm{AP}^{2} \mathrm{R}\right)$ which is approximated to the $\mathrm{P}^{2} \mathrm{R}$ approach. Other contribution work about perspective reformulation can be seen in (Günlük \& Linderoth, 2012; Zheng, Sun, \& Li, 2014).

Besides the perspective reformulation, another type of reformulation method called lift-and-convexification reformulation (LCR) has been put forward in (Wu, Sun, Li, \& Zheng, 2015). The substance of this approach is to add a quadratic equivalent term multiplied by a parameter to the objective function and to convexify the objective function so that the resulting formulation equivalent to the original problem. Moreover, the continuous relaxation of reformulation obtains a lower bound the same as that obtained from the perspective reformulation in (Zheng et al., 2014) with dramatically reduced computational time of (SDP). This approach originates from the idea of quadratic convex reformulation (QCR) method which was first introduced for binary quadratic programming in (Hammer \& Rubin, 1970) to show that by adding a quadratic equivalent term to the objective function, the original problem is equivalent to one convex quadratic programming that has positive semidefinite matrix. Billionnet, Elloumi and Plateau $(2008,2009)$ improved this method further by adding an equality constraint in the reformulation and found the best convex reformulation by semidefinite programming. After that Billionnet, Elloumi and Lambert $(2012,2015)$ extended the QCR method to general mixed-integer programs.

The paper is organized as follows: In section 2, we review the current state-of-the-art perspective reformulation for the MIQP with semicontinuous variables and section 3 gives a review of the recent work LCR for the MIQP with semi-continuous variables. In section 4 , we conduct numerical experiment to compare the effectiveness of perspective reformulation and LCR. Conclusions are made in section 6.

Notation: Throughout the paper, we denote by $v(\cdot)$ the optimal value of problem $(\cdot)$, and $\square^{n}$ the nonnegative orthant of $\square^{n}$. For any $a \in \square^{n}$, we denote by $\operatorname{diag}(a)=\operatorname{diag}\left(a_{1}, \ldots, a_{n}\right)$ the diagonal matrix with $a_{i}$ being the $i$ th diagonal element. For any matrix $A$, we denote $A \geq 0$ as $A$ is a semidefinite matrix in our paper.

\section{Perspective Reformulation Review}

In this section, we make a review of perspective reformulation of mixed-integer quadratic programming with semi-continuous variables and cardinality constraint. Firstly, the perspective reformulation requires the objective functionis separable while the objective function $f(x)$ in $(\mathrm{QP})$ is usually nonseparable. Frangioni and Gentile (2007) proposed a diagonal decomposition method to decompose the quadratic form $x^{T} Q x$ as $x^{T}(Q-\operatorname{diag}(d)) x+$ $x^{T} \operatorname{diag}(d) x$ so that extracting the separable terms, where $d \in \square_{+}^{n}$ with $Q-D \geq 0$ and $\operatorname{diag}(d)$ is a diagonal $n \times n$ matrix with the elements of $d$ on the diagonal. Replacing the separable term $x^{T} \operatorname{diag}(d) x$ with its convex envelope over the semi-continuous variables, which is the sum of the perspective functions of $d_{i} x_{i}^{2}$ over $x_{i} \in\{0\} \cup\left[\alpha_{i}, \beta_{i}\right]$, $i=1, \ldots, n$, then the perspective reformulation of (QP) can be expressed as the following:

$$
\begin{gathered}
(\operatorname{PR}(d)) \quad \min x^{T}(Q-\operatorname{diag}(d))+c^{T} x+\sum_{i=1}^{n} d_{i}\left(x_{i}^{2} / y_{i}\right) \\
\text { s.t. (4), (5), (6), (7) }
\end{gathered}
$$

Since the high nonlinearity of objective function due to the fractional term in perspective function of $(\operatorname{PR}(d))$, efficient solution methods can not be directly applied to solve $(\operatorname{PR}(d))$. Then two tractable reformulation methods were proposed to deal with it. One is the second-order cone programming (SOCP) reformulation proposed in (Aktürk, Atamtürk, \& Gürel, 2009; Günlük, \& Linderoth, 2010) which introducing an additional variable $\phi_{i}=x_{i}^{2} / y_{i}$ for each $i=1, \ldots, n$ and then rewriting the $\phi_{i} \geq x_{i}^{2} / y_{i}$ as an SOCP constraint. The resulting SOCP reformulation of $(\operatorname{PR}(d))$ can be rewrite as the following form:

$$
\begin{aligned}
(\operatorname{SOCP}(d)) & \min x^{T}(Q-\operatorname{diag}(d))+c^{T} x+\phi^{T} d \\
& \text { s.t. } \quad\left\|\begin{array}{l}
x_{i} \\
\phi-y_{i} \\
2
\end{array}\right\| \leq \frac{\phi+y_{i}}{2}, i=1, \ldots, n,
\end{aligned}
$$


(4), (5), (6), (7)

Another is the perspective cut $(\mathrm{P} / \mathrm{C})$ reformulation proposed in (Frangioni and Gentile, 2007). Representing the epigraph of $x_{i}^{2} / y_{i}$ by a set of perspective cut inequalities which can be expressed as the following form:

$$
\begin{aligned}
(\mathrm{PC}(d)) \quad \min & x^{T}(Q-\operatorname{diag}(d))+c^{T} x+\sum_{i=1}^{n} d_{i} v_{i} \\
& \text { s.t. } \quad v_{i} \geq 2 \bar{x}_{i} x_{i}-\bar{x}_{i}^{2} y_{i}, \forall \bar{x}_{i} \in\left[\alpha_{i}, \beta_{i}\right], i=1, \ldots, n,
\end{aligned}
$$

(4), (5), (6), (7)

A key point is how to choose the best parameter vector $d$ when implementing the SOCP reformulation $(\mathrm{SOCP}(d))$ and $(\mathrm{P} / \mathrm{C})$ reformulation $(\mathrm{PC}(d))$ so that the lower bound is as tight as possible. One simple way is to select the smallest eigenvalue of $Q$ as the elements of vector $d$. Then Frangioni and Gentile (2007) proposed a heuristic method to find a "better" $d$ compared with the smallest eigenvalue method by solving the following "small" semidefinite programming (SDP):

$$
\left(\mathrm{SDP}_{s}\right) \quad \max \left\{e^{T} d \mid Q-D \geq 0, d \geqslant 0\right\},
$$

Zheng et al. (2014) proposed a large SDP approach to find the "best" parameter vector $d$ in the perspective reformulation. Since the continuous relaxations $(\overline{\mathrm{PR}}(d)),(\overline{\mathrm{SOCP}}(d)),(\overline{\mathrm{PC}}(d))$ have the same continuous bounds, the best parameter vector $d$ that maximize the $v(\overline{\mathrm{PR}}(d))$ can be found by solving the following problem:

$$
\left(\mathrm{MAX}_{d}\right) \quad \max \{v(\overline{\mathrm{PR}}(d)) \mid Q-D \geq 0, d \geqslant 0\}
$$

According to the conclusion in (Zheng et al., 2014), problem $\left(\mathrm{MAX}_{d}\right)$ is equivalent to the following SDP problem:

$$
\begin{aligned}
\left(\mathrm{SDP}_{l}\right) \quad & \max -K s-e^{T} \pi-\tau \\
\text { s.t. } \quad & \left(\begin{array}{ll}
d_{i}+\mu_{i} & \frac{1}{2}\left(c_{i}-\lambda_{i}-\left(\alpha_{i}+\beta_{i}\right) \mu_{i}\right) \\
\frac{1}{2}\left(c_{i}-\lambda_{i}-\left(\alpha_{i}+\beta_{i}\right) \mu_{i}\right)^{T} & \pi_{i}+s+\alpha_{i} \beta_{i} \mu_{i}
\end{array}\right) \geq 0, \\
& i=1, \ldots, n, \\
& \left(\begin{array}{ll}
Q-\operatorname{diag}(\phi) & \frac{1}{2}\left(\lambda+A^{T} \eta\right) \\
\frac{1}{2}\left(\lambda+A^{T} \eta\right)^{T} & -\eta^{T} b+\tau
\end{array}\right) \geq 0, \\
& (s, \eta, \mu, \pi, d) \in{ }_{+} \times \square{ }_{+}^{m} \times \square{ }_{+}^{n} \times \square_{+}^{n} \times \square_{+}^{n}, \\
& (\tau, \lambda) \in \square \times \square^{n}
\end{aligned}
$$

Although the computation time of solving the $\mathrm{SDP}$ problem $\left(\mathrm{SDP}_{l}\right)$ is longer than solving $\left(\mathrm{SDP}_{s}\right)$ due to the large dimension, the dramatically time reduction in computing SOCP or P/C reformulations pays off the long time consumption in $\left(\mathrm{SDP}_{l}\right)$. Moreover, computational results in (Zheng et al.,2014) were shown that using the parameter vector $d$ computed by the large SDP formulation can considerably improve the performance of the perspective reformulations, largely due to the improvement of the continuous bounds.

\section{Lift-and-Convexification Reformulation (LCR) Review}

Another efficient solution method for mixed-integer quadratic programming with semi-continuous variables and cardinality constraint is the lift-and-convexification reformulation (LCR) proposed by Wu et al. (2015), where the original problem is:

$$
\begin{aligned}
\text { (P) } \quad \min \quad & f(x, y)=x^{T} Q x+c^{T} x+h^{T} y \\
\text { s.t. } & A x+B y \leq d, \\
& \alpha_{\mathrm{i}} y_{i} \leq x_{i} \leq \beta_{i} y_{i}, y_{i} \in\{0,1\}, i=1, \ldots, n
\end{aligned}
$$

The LCR is to add a quadratic equivalent term $\sum_{i=1}^{n}\left(u_{i} x_{i}+v_{i} y_{i}\right)\left(y_{i}-1\right)$, where $u \in \square^{n}, v \in \square^{n}$ to the objective function and to convexify the objective function at the same time so that the resulting formulation equivalent to 
the original problem. The reformulated problem $(\mathrm{P}(u, v))$ can be expressed as following:

$$
\begin{aligned}
(\mathrm{P}(u, v)) \quad \min & f_{u, v}(x, y)=f(x, y)+\sum_{i=1}^{n}\left(u_{i} x_{i}+v_{i} y_{i}\right)\left(y_{i}-1\right) \\
\text { s.t. } \quad(9),(10) &
\end{aligned}
$$

The reformulated problem $(\mathrm{P}(u, v))$ can be solved by the classical branch-and-bound algorithm based on its lower bound, which is the optimal value of its continuous relaxation. Let $u=\left(u_{1}, \ldots, u_{n}\right)^{T}$ and $v=\left(v_{1}, \ldots, v_{n}\right)^{T}$, $(\overline{\mathrm{P}}(u, v))$ denote the continuous relaxation of $(\mathrm{P}(u, v))$ by relaxing $y \in\{0,1\}^{n}$ to $y \in[0,1]^{n}$. Moreover, we define the value of $(\mathrm{P}(u, v))$ as $v(\overline{\mathrm{P}}(u, v))$. The best parameters $(u, v)$ can be found by solving the following problem:

$$
\left(\operatorname{MAX}_{u v}\right) \quad \max \left\{v(\overline{\mathrm{P}}(u, v)) \mid u, v \in \square^{n}, f_{u, v}(x, y) \text { is convex }\right\}
$$

Theorem 1 Problem $\left(M A X_{u v}\right)$ is equivalent to the following semidefinite programming SDP problem:

$$
\begin{aligned}
\left(\mathrm{SDP}_{l}\right) & \max -K s-e^{T} \pi-\tau \\
\text { s.t. } & \left(\begin{array}{lcc}
Q & \frac{1}{2} \operatorname{diag}(u) & \frac{1}{2} \alpha(u, \eta, \mu, \sigma) \\
\frac{1}{2} \operatorname{diag}(u) & \operatorname{diag}(v) & \frac{1}{2} \beta(v, \eta, \mu, \sigma, \lambda, \pi) \\
\frac{1}{2} \alpha(u, \eta, \mu, \sigma)^{T} & \frac{1}{2} \beta(v, \eta, \mu, \sigma, \lambda, \pi)^{T} & -\eta d-e^{T} \pi-\tau
\end{array}\right) \geq 0, \\
& (\eta, \mu, \sigma, \lambda, \pi) \in \square_{+}^{m} \times \square_{+}^{n} \times \square_{+}^{n} \times \square_{+}^{n} \times \square_{+}^{n}, \\
& (u, v, \tau) \in \square^{n} \times \square^{n} \times \square
\end{aligned}
$$

Where

$$
\begin{aligned}
& \alpha(u, \eta, \mu, \sigma)=c-u+A^{T} \eta-\mu+\sigma \\
& \beta(v, \eta, \mu, \sigma, \lambda, \pi)=h-v+B^{T} \eta+\operatorname{diag}(\alpha) \mu-\operatorname{diag}(\beta) \sigma-\lambda+\pi
\end{aligned}
$$

\section{Computational results}

In this section, we conduct a series of computational experiments for the mean-variance portfolio selection problem $(\mathrm{QP})$ with cardinality and minimum threshold constraints in real-life described in section 1 . The aim of our numerical tests is to compare the performance of the perspective reformulation and the LCR proposed in section 2 and section 3 respectively under the branch-and-bound algorithm. Since Frangioni and Gentile (2009) has drew the conclusion that the $(\operatorname{PC}(d))$ is more competitive than the $(\operatorname{SOCP}(d))$ if probably processed, then we focus on the following two reformulation method:

-(PC): the perspective cut reformulation $(\mathrm{PC}(d))$ with $d=d^{*}$, where $d^{*}$ is obtained by solving $\left(\mathrm{SDP}_{l}\right)$.

-(LCR): the lift-and-convexification reformulation $(\mathrm{P}(u, v))$ with $(u, v)=\left(u^{*}, v^{*}\right)$, where $\left(u^{*}, v^{*}\right)$ is obtained by solving $\left(\mathrm{SDP}_{q}\right)$.

To conduct the test of our approach for above MV problem, we randomly generated 5 instances for each test problem with the same size $(n=200,300,400)$. For each instance, test was conducted under $K=4,6,8,12$ and without cardinality constraint. The diagonal elements and non diagonal elements of the real symmetric matrix $Q$ are randomly generated in the interval $[4,1000]$ and $[1,10]$, respectively. The elements of matrices $\mu$ and the interval of expected return level $\rho$ are all randomly set in $[0.002,0.01]$. The intervals of minimum and maximum buy-in thresholds $\alpha_{i}$ and $\beta_{i}$ have been randomly set in [0.075, 0.125] and [0.375, 0.425], respectively. The two SDP are all implemented in Matlab R2016b and run on a PC (2.5GHz, 8GB RAM). The computational results are interface as the lower bounds used in CPLEX 12.7 where the MIQP are solved by the MIQP solver. The CPU time limit is set at 10000 seconds and the CPLEX 12.7 is operated with the default setting.

Table 1 reports the computational results of two reformulation methods (PC and LCR) for the MV problem where the column "time" is the computational time for solving $\left(\mathrm{SDP}_{l}\right)$ and "time" $e_{q}$ is the computational time for solving $\left(\mathrm{SDP}_{q}\right)$. Each line reports the average results for the 5 instances in a subset. The column "gap" refers to the relative gap between the objective value of the exiting solution and the best lower bound which is expressed in percentage. The column "time" is the computing time (in seconds) and the column "nodes" is the number of nodes when solved in CPLEX. The "nonK" is expressed as the instances without cardinality constraint. 
Table 1. Comparison Results of Reformulations for (MV)

\begin{tabular}{|c|c|c|c|c|c|c|c|c|c|}
\hline \multirow{2}{*}{ (MV) } & \multirow{2}{*}{ K } & \multirow{2}{*}{ time $_{l}$} & \multirow{2}{*}{ time $_{q}$} & \multicolumn{3}{|c|}{ (PC) } & \multicolumn{3}{|c|}{ (LCR) } \\
\hline & & & & gap & time & nodes & gap & time & nodes \\
\hline \multirow{5}{*}{200} & 4 & 15.56 & 46.63 & 0 & 5.86 & 117 & 0 & 2.75 & 105 \\
\hline & 6 & 12.33 & 41.86 & 0 & 1.98 & 50 & 0 & 1.19 & 38 \\
\hline & 8 & 11.2 & 41.22 & 0 & 2.19 & 60 & 0 & 1.25 & 62 \\
\hline & 12 & 10.84 & 51.75 & 0 & 1.69 & 49 & 0 & 1.13 & 44 \\
\hline & nonK & 12.83 & 42.47 & 0 & 1.20 & 56 & 0 & 1.11 & 84 \\
\hline \multirow{5}{*}{300} & 4 & 24.45 & 144.69 & 0 & 53.72 & 492 & 0 & 60.57 & 541 \\
\hline & 6 & 29.02 & 159.55 & 0 & 7.27 & 187 & 0 & 5.5 & 223 \\
\hline & 8 & 26.36 & 167.44 & 0 & 6.88 & 73 & 0 & 5.32 & 83 \\
\hline & 12 & 23.83 & 165.81 & 0 & 11.41 & 200 & 0 & 20.16 & 270 \\
\hline & nonk & 24.45 & 179.31 & 0 & 5.87 & 112 & 0 & 15.26 & 190 \\
\hline \multirow{5}{*}{400} & 4 & 53.31 & 457.13 & 0 & 1234.85 & 3038 & 0 & 1411.56 & 11353 \\
\hline & 6 & 52.03 & 427.92 & 0 & 191.7 & 1126 & 0 & 233.07 & 1990 \\
\hline & 8 & 61.19 & 514.03 & 0 & 229.06 & 3521 & 0 & 848.92 & 9831 \\
\hline & 12 & 60.73 & 460.59 & 0 & 158.28 & 926 & 0 & 695.63 & 4629 \\
\hline & nonk & 58.83 & 383.45 & 0 & 40.54 & 435 & 0 & 59.74 & 604 \\
\hline
\end{tabular}

From the Table 1 we can see that the average computing time of $\left(\mathrm{SDP}_{l}\right)$ is far less than the computing time of $\left(\mathrm{SDP}_{q}\right)$, what's more, the computing time gap between $\left(\mathrm{SDP}_{l}\right)$ and $\left(\mathrm{SDP}_{q}\right)$ increases dramatically as the dimension increases. On the other hand, when comparing the time of MIQP solver CPLEX, the computing time for (PC) is longer than (LCR) as $n=200$. However, the computing time for (PC) is less than (LCR) when $n=$ 300,400 . From the data in column "gap", we can see that the lower bound obtained from $\left(\mathrm{SDP}_{l}\right)$ and $\left(\mathrm{SDP}_{q}\right)$ is nearly the same as the objective value obtained by CPLEX 12.7. Moreover, for the total computing time (the sum of time for solving SDP and corresponding PC/LCR), (PC) performs better than (LCR) for all the instances. For the number of nodes explored in (PC) and (LCR), (PC) performs better than (LCR) for 12 out of the total 15 instances. The computational result is well as we expected because the relaxations in (LCR) are in general looser than the ones in (PC) at children nodes.

\section{Conclusions}

We have reviewed two kinds of reformulation methods for the mean-variance portfolio selection problem with cardinality and minimum buy-in threshold constraints which can be classified as the mixed-integer quadratic programming problems (MIQP). One is Perspective Reformulation and another is Lift-and-Convexification (LCR). We mainly focus on the theory review and its application in portfolio selection problems. According to the computational results, both the (PC) and the (LCR) are useful tools for obtaining the tighter lower bounds on MIQP with semi-continuous variables and cardinality constraint. Further more, it seems that the (PC) is more competitive than the (LCR) method in both computing time and nodes in MIQP solver CPLEX 12.7 and this outperformance becomes more obvious as the size of instances grows.

\section{References}

Aktürk, M. S., Atamtürk, A., \& Gürel, S. (2009). A strong conic quadratic reformulation for machine-job assignment with controllable processing times. Operations Research Letters, 37(3), 187-191. https://doi.org/10.1016/j.orl.2008.12.009

Andersson, F., Mausser, H., Rosen, D., \& Uryasev, S. (2001). Credit risk optimization with conditional value-at -risk criterion. Mathematical Programming, 89(2), 273-291. https://doi.org/10.1007/PL00011399

Bertsimas, D., \& Shioda, R. (2009). Algorithm for cardinality-constrained quadratic optimization. Computational Optimization and Applications, 43(1), 1-22. https://doi.org/10.1007/s10589-007-9126-9

Bienstock, D. (1996). Computational study of a family of mixed-integer quadratic programming problems. Mathematical Programming, 74(2), 121-140. https://doi.org/10.1007/BF02592208 
Billionnet, A., Elloumi, S., \& Lambert, A. (2012). Extending the QCR method to general mixed-integer programs. Mathematical programming, 131(1-2), 381-401. https://doi.org/10.1007/s10107-010-0381-7

Billionnet, A., Elloumi, S., \& Lambert, A. (2015). A branch and bound algorithm for general mixed-integer quadratic programs based on quadratic convex relaxation. Journal of Combinatorial Optimization, 28(2), 376-399. https://doi.org/10.1007/s10878-012-9560-1

Billionnet, A., Elloumi, S., \& Plateau, M.-C. (2008). Quadratic 0-1 programming: tightening linear or quadratic convex reformulation by use of relaxations. RAIRO-Operations Research, 42(2), 103-121. https://doi.org/10.1051/ro:2008011

Billionnet, A., Elloumi, S., \& Plateau, M.-C. (2009). Improving the performance of standard solvers for quadratic 0-1 programs by a tight convex reformulation: The QCR method. Discrete Applied Mathematics, 157(6), 1185-1197. https://doi.org/10.1016/j.dam.2007.12.007

Blog, B., Hoek, G. V. D., \& Timmer, G. T. (1983). The optimal selection of small portfolios. Management Science, 29(7), 792-798. https://doi.org/10.1287/mnsc.29.7.792

Bonami, P., \& Lejeune, M. A. (2009). An exact solution approach for portfolio optimization problems under stochastic and integer constraints. Operations research, 57(3), 650-670. https://doi.org/10.1287/opre.1080.0599

Cesarone, F., Scozzari, A., \& Tardella, F. (2009). Efficient algorithms for mean-variance portfolio optimization with hard real-world constraints. Giornale dell'Istituto Italiano degli Attuari, 72, 37-56.

Chang, T. J., Meade, N., Beasley, J. E., \& Sharaiha, Y. M. (2000). Heuristics for cardinality constrained portfolio optimisation. Computers and Operations Research, 27(13), 1271-1302. https://doi.org/10.1016/S0305-0548(99)00074-X

Cui, X. T., Zheng, X. J., Zhu, S. S., \& Sun, X. L. (2013). Convex relaxations and MIQCQP reformulations for a class of cardinality-constrained portfolio selection problems. Journal of Global Optimization, 56(4), 1409-1423. https://doi.org/10.1007/s10898-012-9842-2

Frangioni, A., Furini, F., \& Gentile, C. (2016). Approximated perspective relaxations: a project and lift approach. Computational Optimization and Applications, 63(3), 705-735. https://doi.org/10.1007/s10589-015-9787-8

Frangioni, A., \& Gentile, C. (2006). Perspective cuts for a class of convex 0C1 mixed integer programs. Mathematical Programming, 106(2), 225-236. https://doi.org/10.1007/s10107-005-0594-3

Frangioni, A., \& Gentile, C. (2007). SDP diagonalizations and perspective cuts for a class of nonseparable MIQP. Operations Research Letters, 35(2), 181-185. https://doi.org/10.1016/j.orl.2006.03.008

Frangioni, A., \& Gentile, C. (2009). A computational comparison of reformulations of the perspective relaxation: SOCP vs. cutting planes. Operations Research Letters, 37(3), 206-210. https://doi.org/10.1016/j.orl.2009.02.003

Frangioni, A., Gentile, C., Grande, E., \& Pacifici, A. (2011). Projected perspective reformulations with applications in design problems. Operations Research, 59(5), 1225-1232. https://doi.org/10.1287/opre.1110.0930

Gao, J., \& Li, D. (2013). Optimal cardinality constrained portfolio selection. Operations Research, 61(3), 745-761. https://doi.org/10.1287/opre.2013.1170

Günlük, O., \& Linderoth, J. (2010). Perspective reformulations of mixed integer nonlinear programs with indicator variables. Mathematical programming, 124(1-2), 183-205. https://doi.org/10.1007/s10107-010-0360-z

Günlük, O., \& Linderoth, J. (2012). Perspective reformulation and applications. https://doi.org/10.1007/s10107-010-0360-z

Green, R. C., \& Hollifield, B. (1992). When will mean-variance efficient portfolios be well diversified? The Journal of Finance, 47(5), 1785-1809. https://doi.org/10.1111/j.1540-6261.1992.tb04683.x

Hammer, P. L., \& Rubin, A. A. (1970). Some remarks on quadratic programming with 0-1 variables. Revue française dinformatique et de recherche opérationnelle. Série verte, 4(V3), 67-79. Retrieved from http://www.numdam.org/article/RO_1970_4_3_67_0.pdf

Jacob, N. L. (1974). A limited-diversification portfolio selection model for the small investor. Journal of Finance, 29(3), 847-856. https://doi.org/10.1111/j.1540-6261.1974.tb01487.x 
Konno, H., Shirakawa, H., \& Yamazaki, H. (1993). A mean-absolute deviation-skewness portfolio optimization model. Annals of Operations Research, 45(1), 205-220. https://doi.org/10.1007/BF02282050

Konno, H., \& Suzuki, K.-i. (1995). A mean-variance-skewness portfolio optimization model. Journal of the Operations Research Society of Japan, 38(2), 173-187. http://doi.org/10.15807/jorsj.38.173

Konno, H., \& Yamazaki, H. (1991). Mean-absolute deviation portfolio optimization model and its applications to Tokyo stock market. Management science, 37(5), 519-531. https://doi.org/10.1287/mnsc.37.5.519

Li, D., Sun, X., \& Wang, J. (2006). Optimal lot solution to cardinality constrained mean-variance formulation for portfolio selection. Mathematical Finance, 16(1), 83-101. https://doi.org/10.1111/j.1467-9965.2006.00262.x

Mansini, R., Ogryczak, W., \& Grazia Speranza, M. (2003). Lp solvable models for portfolio optimization: A classification and computational comparison. IMA Journal of Management Mathematics, 14(3), 187-220. https://doi.org/10.1093/imaman/14.3.187

Mao, J. C. (1970). Models of capital budgeting, Ev vs Es. Journal of financial and quantitative analysis, 4(5), 657-675. https://doi.org/10.2307/2330119

Maringer, D., \& Kellerer, H. (2003). Optimization of cardinality constrained portfolios with a hybrid local search algorithm. OR Spectrum, 25(4), 481-495. https://doi.org/10.1007/s00291-003-0139-1

Markowitz, H. (1952). Portfolio selection. The journal of finance, 7(1), 77-91. https://doi.org/10.1111/j.1540-6261.1952.tb01525.x

Markowitz, H. (1959). Portfolio selection: efficient diversification of investments.

Mitra, G., Ellison, F., \& Scowcroft, A. (2007). Quadratic programming for portfolio planning: Insights into algorithmic and computational issues part ii? processing of portfolio planning models with discrete constraints. Journal of Asset Management, 8(4), 249-258. https://doi.org/10.1057/palgrave.jam.2250079

Ogryczak, W., \& Ruszczynski, A. (1997). On stochastic dominance and mean-semideviation models. Working Papers, 3(3), 169-190. Retrieved from http://pure.iiasa.ac.at/5251/1/IR-97-043.pdf

Ouederni, B. N., \& Sullivan, W. G. (1991). A semi-variance model for incorporating risk into capital investment analysis. The Engineering Economist, 36(2), 83-106. http://doi.org/10.1080/00137919108903035

Rockafellar, R. T., \& Uryasev, S. (2000). Optimization of conditional value-at-risk. Journal of risk, 2, 21-42. https://doi.org/10.21314/JOR.2000.038

Rockafellar, R. T., \& Uryasev, S. (2002). Conditional value-at-risk for general loss distributions. Journal of banking and finance, 26(7), 1443-1471. https://doi.org/10.1016/S0378-4266(02)00271-6

Topaloglou, N., Vladimirou, H., \& Zenios, S. A. (2002). CVaR models with selective hedging for international asset allocation. Journal of Banking and Finance, 26(7), 1535-1561. https://doi.org/10.1016/S0378-4266(02)00289-3

Uryasev, S. (2000). Conditional value-at-risk: Optimization algorithms and applications. Computational intelligence for financial engineering, 49-57. https://doi.org/10.1109/CIFER.2000.844598

Wu, B., Sun, X., Li, D., \& Zheng, X. (2015). Quadratic Convex Reformulations for Semicontinuous Quadratic Programming. Siam Journal on Optimization, 27(3), 1531-1553. https://doi.org/10.1137/15M1012232

Zheng, X., Sun, X., \& Li, D. (2014). Improving the performance of MIQP solvers for quadratic programs with cardinality and minimum threshold constraints: A semidefinite program approach. Informs Journal on Computing, 26(4), 690-703. https://doi.org/10.1287/ijoc.2014.0592

\section{Copyrights}

Copyright for this article is retained by the author(s), with first publication rights granted to the journal.

This is an open-access article distributed under the terms and conditions of the Creative Commons Attribution license (http://creativecommons.org/licenses/by/4.0/). 